\title{
FOUR-SIDED LID-DRIVEN CAVITY FLOW USING TIME SPLITTING METHOD OF ADAMS-BASHFORTH SCHEME
}

\author{
C. S. N. Azwadi", A. Rajab and A. Sofianuddin \\ Faculty of Mechanical Engineering, Universiti Teknologi Malaysia \\ 81310 Skudai, Johor, Malaysia \\ *Email: azwadi@fkm.utm.my \\ Phone: +607-5534718; Fax: +607-5566159
}

\begin{abstract}
In this paper, a second order Adams-Bashforth method is proposed to simulate foursided square lid-driven cavity flow. The convective term and diffusive term in NavierStokes equations are solved using a finite central difference scheme and the unsteady term is solved using the Adams-Bashforth method. All of the flow is simulated at below critical Reynolds numbers in a square cavity with an isothermal condition and the same speed for all walls. In this study, the flow structure for a four-sided lid-driven cavity with its four vortices and a graph of the velocity profile along the center of the cavity are presented. In addition, we also study the effect of the Reynolds number on the development of vortices in the cavity. We find that the Reynolds number has a dominant effect on the flow structure in the cavity. The computed results also show good agreement with the published data.
\end{abstract}

Keywords: Four-sided lid-driven cavity; Adams-Bashforth Method; Reynolds numbers, time splitting method.

\section{INTRODUCTION}

The flow inside a square cavity has been widely used to demonstrate fluid flow using simple geometry. The flow structure in a one-sided lid-driven cavity problem has been reported by many researchers (Ghia, Ghia, \& Shin, 1982; Idris, Ammar, Tuan Ya, \& Amin, 2013; Idris, Irwan, \& Ammar, 2012; Musa, Abdullah, Azwadi, \& Zulkifli, 2011; Rosdzimin, Zuhairi, \& Azwadi, 2010; Sidik \& Attarzadeh, 2011). Later, research on different cavity geometries has been reported, such as a deep cavity (Patil, Lakshmisha, \& Rogg, 2006), semi-circular cavity (Yang, Shi, Guo, \& Sai, 2012), trapezoidal cavity (Zhang, Shi, \& Chai, 2010) and triangular cavity (González, Ahmed, Kühnen, Kuhlmann, \& Theofilis, 2011). Recently, two-sided lid-driven cavity flow (Mendu \& Das, 2012; Oueslati, Ben Beya, \& Lili, 2011) has been analyzed in order to study possible applications in industry, such as in the paper coating industry (Cao \& Esmail, 1995), polymer processing (Gaskell, Summers, Thompson, \& Savage, 1996) and drying technologies (Alleborn, Raszillier, \& Durst, 1999). For the case of a foursided lid-driven cavity flow, Wahba (2009) studied the bifurcation of the flow and found that four-sided lid-driven cavity flow generates bifurcation at $\operatorname{Re}=219$. Recently, Perumal and Dass (2011) and Cadou, Guevel, and Girault (2012) tested the stability of the Lattice Boltzmann method by simulating the flow structure in a four-sided lid-driven cavity. In order to solve the Navier-Stokes equation without having a checkerboard problem, a staggered grid arrangement should be used, where pressure will be located at 
the center of the staggered grid and velocity will be placed at the cell face. The AdamsBashforth method is a famous method that implements a staggered grid for time advancement calculation. A higher order of accuracy analysis for the Adams-Bashforth method has been applied by many researchers, such as Man and Tsai (2008) in their work on the vorticity-stream function equation, as well as work by Lo, Murugesan, and Young (2005). A similar formulation has been used, combining the Adams-Bashforth scheme with the Crank-Nicolson scheme to create a semi-implicit scheme in threedimensional analysis (Hansen, Sørensen, \& Shen, 2003). To solve the AdamsBashforth scheme, a method called the time-splitting method needs to be used to reduce the number of variables in the momentum equation. Such work has already been done by many researchers (Boersma, 2011; Choi \& Balaras, 2009; Perot, 1993).

It appears from the aforementioned investigations that numerous investigations have been conducted on the lid-driven cavity flow. However, no attempt has been made to investigate four-sided lid-driven cavity flow with a high order and stable numerical scheme such as the Adams-Bashforth Crank-Nicolson scheme. Different values of Reynolds number such as at 10,100 and 127 will be reported. This study is focused on a low Reynolds number as the critical Reynolds number for the four-sided cavity problem is 129 (Wahba, 2009). In the present study, the top wall is moving to the right, the bottom wall is moving to the left, the right wall is moving upwards and the left wall is moving downwards with equal speed. Before presenting the results, the validation of codes will be presented at $\operatorname{Re}=1000$, with the streamline patterns on a four-sided liddriven cavity, together with the location of the vortices, and a comparison with the work of Perumal and Dass (2011). Later, their normalized velocity along the center of the cavity will be presented.

\section{MATHEMATICAL MODEL}

The incompressible Navier-Stokes equations and continuity equation are written in nondimensional form. The flow is considered as incompressible and in an isothermal condition.

Continuity equation:

$$
\frac{\partial U}{\partial X}+\frac{\partial V}{\partial Y}=0
$$

Momentum equation:

$$
\begin{aligned}
& \frac{\partial U}{\partial T}+\frac{\partial U U}{\partial X}+\frac{\partial U V}{\partial Y}=-\frac{\partial P}{\partial X}+\frac{1}{R e}\left(\frac{\partial^{2} U}{\partial X^{2}}+\frac{\partial^{2} U}{\partial Y^{2}}\right) \\
& \frac{\partial V}{\partial T}+\frac{\partial V U}{\partial X}+\frac{\partial V V}{\partial Y}=-\frac{\partial P}{\partial Y}+\frac{1}{R e}\left(\frac{\partial^{2} V}{\partial X^{2}}+\frac{\partial^{2} V}{\partial Y^{2}}\right)
\end{aligned}
$$

where $R e=U L / v$ is the Reynolds number and

$U=$ speed of the wall

$L=$ length of the wall

$v=$ dynamic viscosity of the fluid inside the cavity 
These equations are solved by using the central difference on the convective and diffusive term to get better accuracy. To avoid a checkerboard problem from using the central difference, a staggered grid arrangement is used in this study. For time advancement using the Adams-Bashforth method, the popular method used to remove the pressure term is the time-splitting method. This method is also called the pressure correction method, projection approach, fractional step method or time stepping method. The principle of this method is to remove the pressure term from the equation and to introduce an intermediate velocity. A second order Adams-Bashforth method is used and the equation can be written as

$$
\widehat{U}=U^{n}+\frac{1}{2} \Delta T\left(3 H^{n}-H^{n-1}\right)
$$

In this equation, $H$ represents the convective term with a viscous term in the momentum equation and intermediate velocity will be solved first. In this calculation, the viscous and convective term are discretized by using the central difference. All of the velocity component that is not located exactly on the grid will be calculated by using averaging, as will be discussed later in the boundary condition section. During the intermediate step, the pressure term will be decoupled in the equation. Pressure correction will then be calculated by using the pressure Poisson equation in Eq. (5), using the intermediate velocity earlier. The pressure should then be used to get the corrected velocity at time step $n+1$ by including the intermediate velocity using Eq. (6).

$$
\begin{gathered}
\frac{\nabla \cdot \widehat{U}}{\Delta T}=\nabla^{2} P^{\mathrm{n}+1} \\
U^{n+1}=\widehat{U}-\frac{\Delta T}{\Delta X}\left(\Delta P^{\mathrm{n}+1}\right)
\end{gathered}
$$

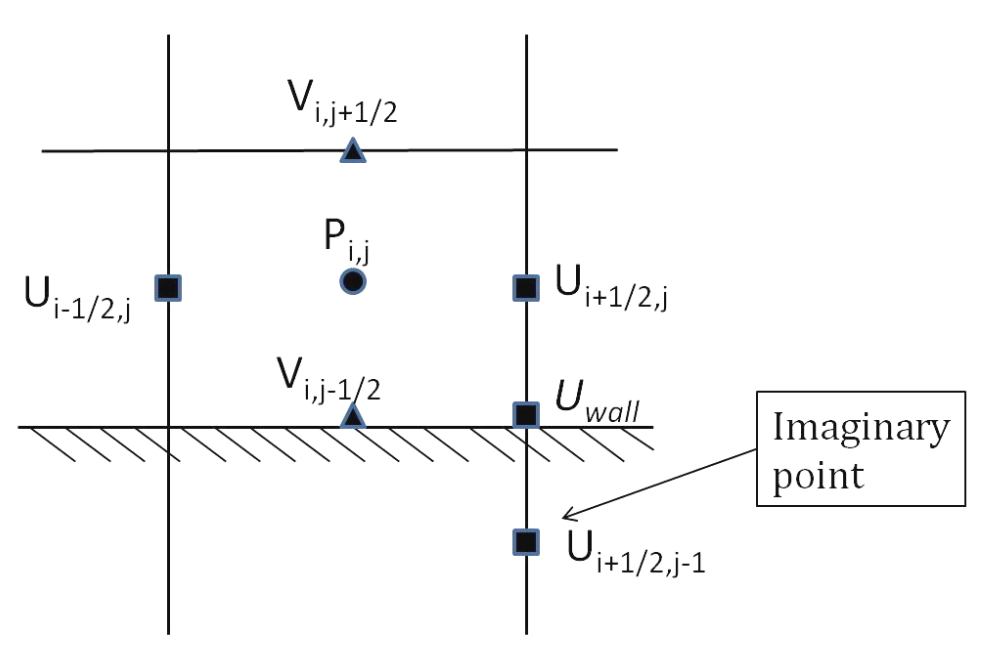

Figure 1. Staggered boundary condition for U-velocity at horizontal wall.

For the horizontal wall, the boundary condition for v-velocity (vertical velocity) can be used directly because the point of vertical velocity is located at the staggered grid, as in Figure 1. But for u-velocity (horizontal velocity), the velocity is not located exactly on the wall and averaging is therefore used at that position. The procedure is the same at the top wall, but the velocity at the top wall is not zero. 


$$
\begin{gathered}
U_{\text {wall }}=\frac{U_{i+\frac{1}{2}, j}+U_{i+\frac{1}{2}, j-1}}{2} \\
U_{i+\frac{1}{2}, j-1}=2 U_{\text {wall }}-U_{i+\frac{1}{2}, j}
\end{gathered}
$$

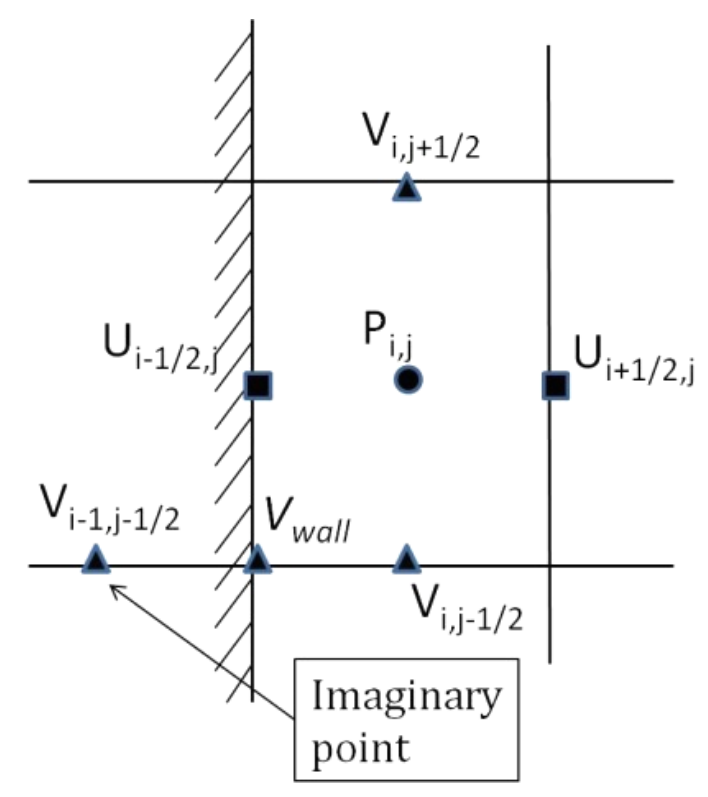

Figure 2. Staggered boundary condition for v-velocity at vertical wall.

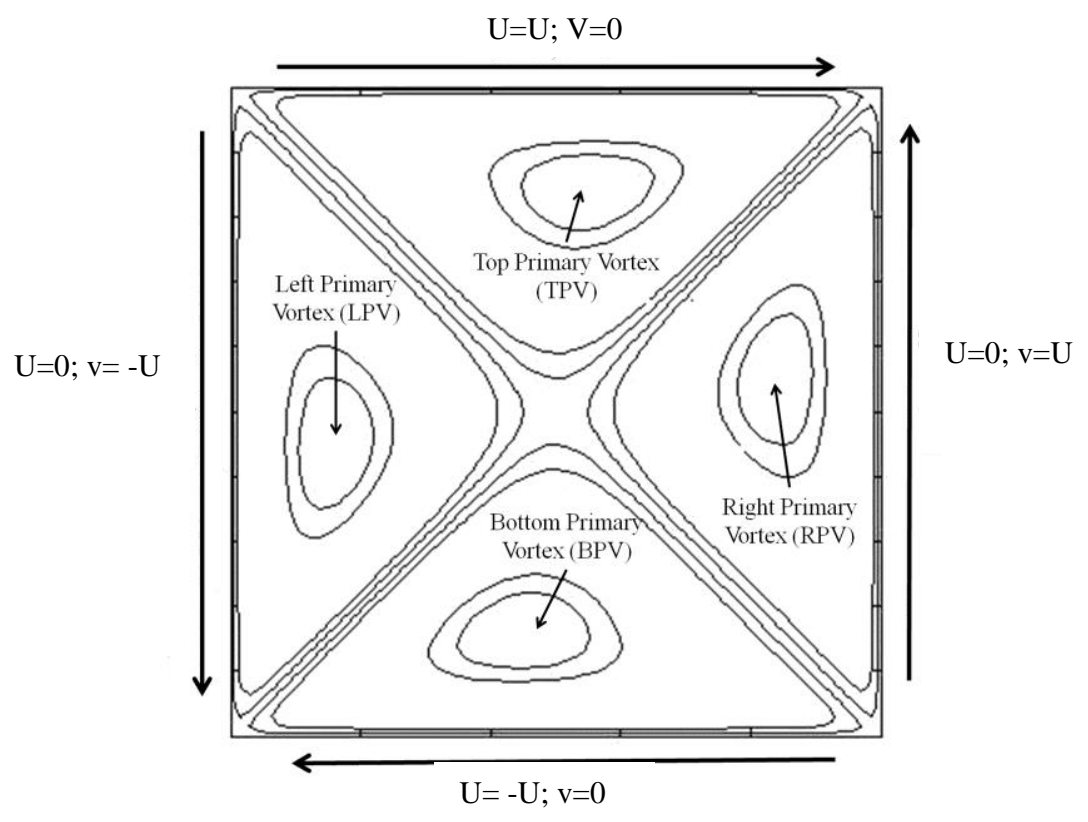

Figure 3. Boundary condition and names of vortices in a four-sided lid-driven cavity flow.

Figure 2 shows the boundary condition for v-velocity at the vertical wall. The boundary condition for u-velocity (horizontal velocity) is now different at the vertical wall, where its node is located on the vertical wall, so it can be used directly. The 
procedure for averaging the v-velocity is the same as in Eq. (7). The walls of the square cavity are moved at the same speed. The top wall moves to the right but the bottom wall moves to the left. On the other hand, the right wall is moving upwards but the left wall is moving downwards, as in Figure 3. Four vortices will developed, where the top vortex is called the Top Primary Vortex (TPV), the vortex at the bottom of the cavity is the Bottom Primary Vortex (BPV), and the right and the left vortices are called the Right Primary Vortex (RPV) and Left Primary Vortex (LPV) respectively. The simulation was done using MATLAB and the grid size is $161 \times 161$ in a staggered grid arrangement.

\section{VALIDATION}

Ghia et al. (1982) used a vorticity-stream function formulation to simulate the flow of a one-sided lid-driven cavity from low to high Reynolds number. Figure 4(a) shows a comparison of the v-velocity profile along the center of the cavity and Figure 4(b) shows the u-velocity. It shows good agreement with the reference.

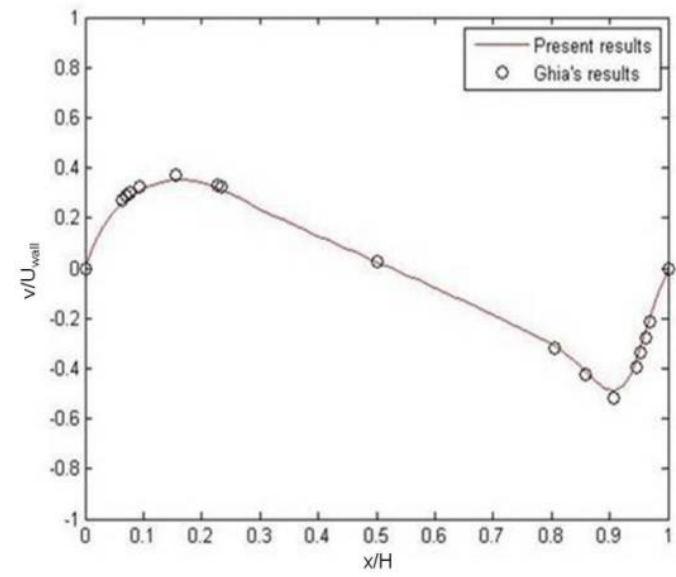

(a)

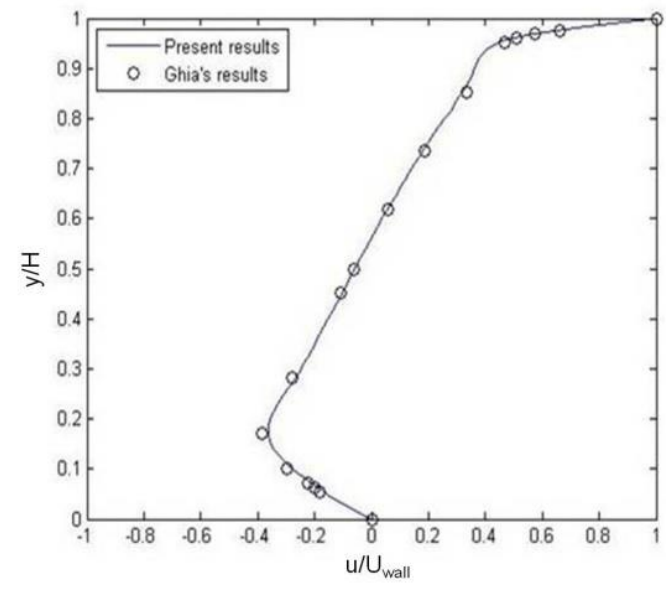

(b)

Figure 4. Validation of current study against Ghia et al. (1982): (a) graph of v-velocity profile along $\mathrm{y}=0.5$ and (b) graph of $\mathrm{u}$-velocity profile along $\mathrm{x}=0.5$ inside cavity.

\section{RESULTS AND DISCUSSION}

This section presents the numerical results of a four-sided lid-driven cavity at $\operatorname{Re}=10$, 100 and 127. The streamlines inside the cavity, the locations of the vortices, and the graph of the velocity profile along the $\mathrm{x}$-axis and $\mathrm{y}$-axis will be discussed. Figure 5 shows the streamline patterns of the four-sided lid-driven cavity: (a) for $\operatorname{Re}=10$, (b) $\operatorname{Re}$ $=100$ and (c) $\operatorname{Re}=127$. Four vortices are generated at the end of the simulation, namely, one vortex each at the top of the cavity, bottom of the cavity, left side of the cavity and right side of the cavity. Generally, the centers of the vortices are seen coming closer to the diagonal joining edges of the moving wall as the Reynolds number increases. Table 1 shows the location of the centers of the vortices for the four-sided liddriven cavity flow at various Reynolds numbers. In general, the vortices move nearer to the diagonal joining edge of the cavity as the Reynolds numbers increase. For comparison of the locations of the vortices from $\operatorname{Re}=10$ to $\operatorname{Re}=100$, the TPV is moving to the right and slightly downwards which is at $(0.559,0.845)$ and the RPV is 
moving upwards and slightly to the left at $(0.845,0.559)$. On the other hand, the BPV and LPV are moving in opposite directions to the TPV and RPV respectively.

Table 1. Locations of vortices centers for four-sided lid-driven cavity flow at various Reynolds numbers.

\begin{tabular}{ccccc}
\hline $\begin{array}{c}\text { Reynolds } \\
\text { number }\end{array}$ & $\begin{array}{c}\text { Left Primary } \\
\text { Vortex (LPV) }\end{array}$ & $\begin{array}{c}\text { Right Primary } \\
\text { Vortex (RPV) }\end{array}$ & $\begin{array}{c}\text { Top Primary } \\
\text { Vortex (TPV) }\end{array}$ & $\begin{array}{c}\text { Bottom Primary } \\
\text { Vortex (BPV) }\end{array}$ \\
\hline 10 & $0.155,0.497$ & $0.851,0.509$ & $0.509,0.851$ & $0.497,0.155$ \\
100 & $0.161,0.442$ & $0.845,0.559$ & $0.559,0.845$ & $0.442,0.161$ \\
127 & $0.168,0.442$ & $0.839,0.559$ & $0.559,0.839$ & $0.442,0.168$ \\
\hline
\end{tabular}

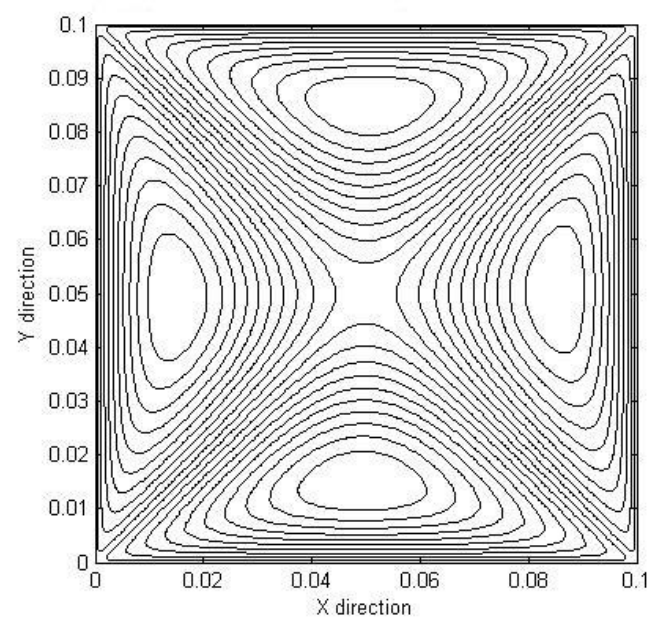

(a)

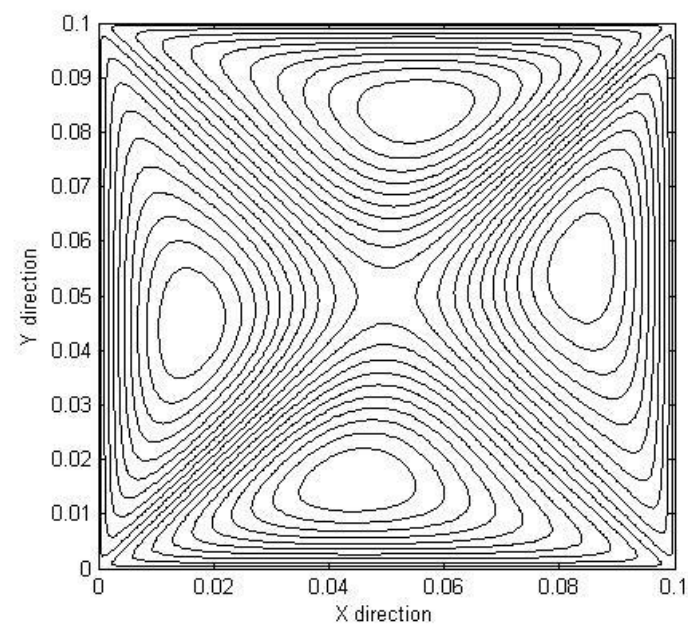

(b)

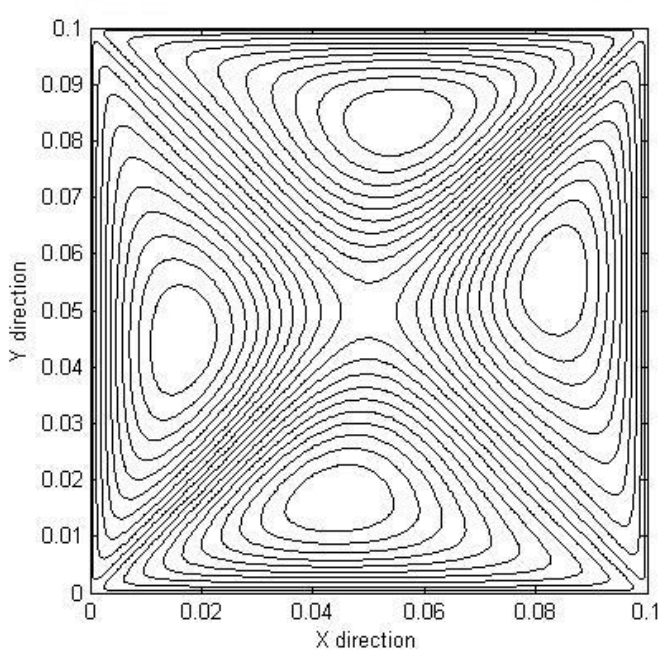

(c)

Figure 5. Streamline patterns for four-sided lid-driven cavity: (a) $\operatorname{Re}=10$, (b) $\operatorname{Re}=100$ and (c) $\operatorname{Re}=127$.

Table 2 shows the values of the dimensionless streamfunction at the centers of the vortices at various Reynolds numbers. The table shows that at certain Reynolds 
numbers, the streamfunction values are the same in magnitude but may have positive or negative values. A negative value indicates that the vortex is moving in a clockwise direction, while a positive value indicates the anti-clockwise direction. The streamfunction values at the TPV are $-0.06553,-0.07046$ and -0.07273 for $\operatorname{Re}=10, \operatorname{Re}=$ 100 and $\operatorname{Re}=127$ respectively. This shows that the magnitude increases as the Reynolds numbers increase.

Table 2. Dimensionless streamfunction values at centers of vortices at various Reynolds numbers.

\begin{tabular}{ccccc}
\hline $\begin{array}{c}\text { Reynolds } \\
\text { number }\end{array}$ & $\begin{array}{c}\text { Left Primary } \\
\text { Vortex (LPV) }\end{array}$ & $\begin{array}{c}\text { Right Primary } \\
\text { Vortex (RPV) }\end{array}$ & $\begin{array}{c}\text { Top Primary } \\
\text { Vortex (TPV) }\end{array}$ & $\begin{array}{c}\text { Bottom Primary } \\
\text { Vortex (BPV) }\end{array}$ \\
\hline 10 & 0.06553 & 0.06553 & -0.06553 & -0.06553 \\
100 & 0.07046 & 0.07046 & -0.07046 & -0.07046 \\
127 & 0.07273 & 0.07273 & -0.07273 & -0.07273 \\
\hline
\end{tabular}

Figure 6 shows the velocity profile along the centerline of the cavity for $\operatorname{Re}=10$, 100 and 127. Generally, it can be said that as the Reynolds number increases, the pattern of the profile is the almost the same, with only a slight quantitative difference. The maximum velocity magnitude is at the wall of the cavity due to the movement. As we move to the center, the magnitude and direction of the velocity change as the flow circulation happens. The present work is then compared to Perumal and Dass (2011) with respect to the centers of the vortices, and shows good agreement. Table 3 shows a comparison of the centers of the vortices at TPV, BPV, LPV and RPV from the present work with Perumal and Dass (2011) at various Reynolds numbers. For the whole range of Reynolds numbers, the locations of the vortices show good comparison results.

Table 3.Comparison of centers of vortices at LPV and RPV of present work with Perumal and Dass (2011) at various Reynolds numbers.

\begin{tabular}{lccccc}
\hline $\begin{array}{c}\text { Reynolds } \\
\text { number }\end{array}$ & Reference & TPV & BPV & LPV & RPV \\
\hline \multirow{3}{*}{$\operatorname{Re}=10$} & Present work & 0.509, & 0.497, & $0.155,0.497$ & 0.851, \\
& 0.851 & 0.155 & & 0.509 \\
& Perumal and Dass & 0.510, & 0.490, & $0.150,0.490$ & 0.849, \\
& $(2011)$ & 0.850 & 0.149 & & 0.510 \\
$\operatorname{Re}=$ & Present work & 0.559, & 0.442, & $0.161,0.442$ & 0.845, \\
100 & 0.845 & 0.161 & & 0.559 \\
& Perumal and Dass & 0.550, & 0.450, & $0.160,0.450$ & 0.840, \\
& $(2011)$ & 0.840 & 0.160 & & 0.550 \\
$\operatorname{Re}=$ & Present work & 0.559, & 0.442, & $0.168,0.442$ & 0.839, \\
127 & (Perumal \& Dass, & 0.839 & 0.168 & & 0.559 \\
& $2011)$ & 0.830 & 0.449, & $0.170,0.450$ & 0.830, \\
& & & & & 0.550 \\
\hline
\end{tabular}




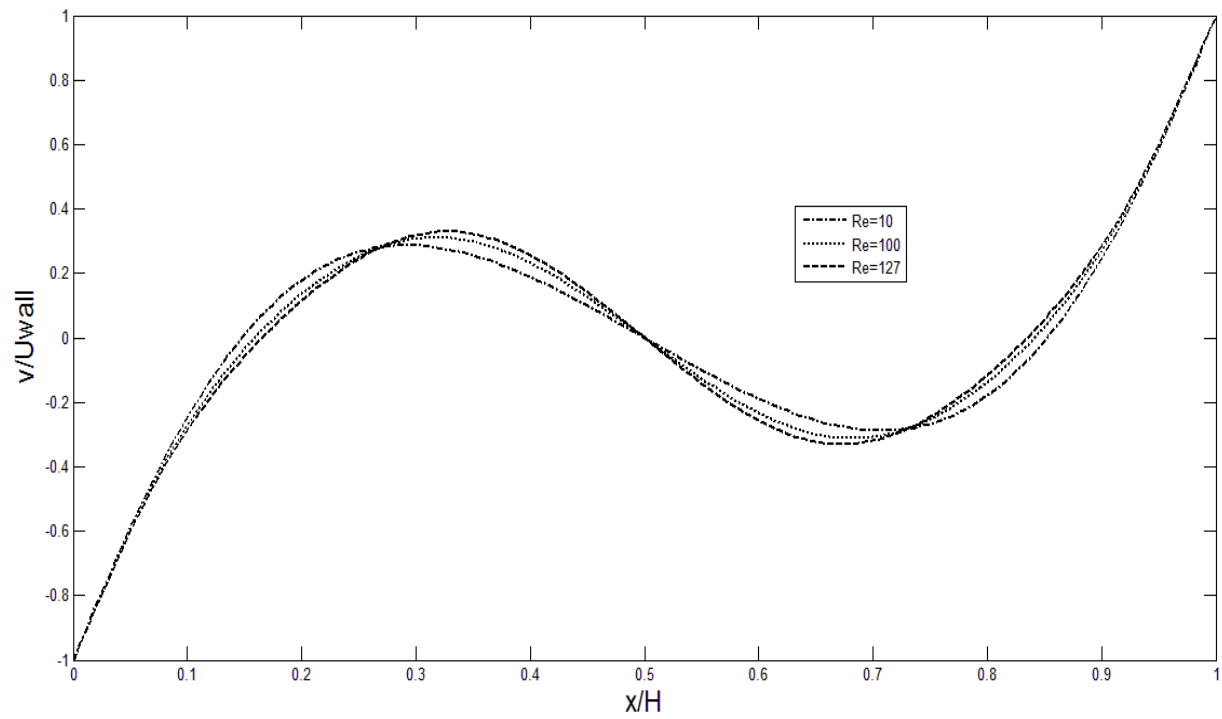

(a)

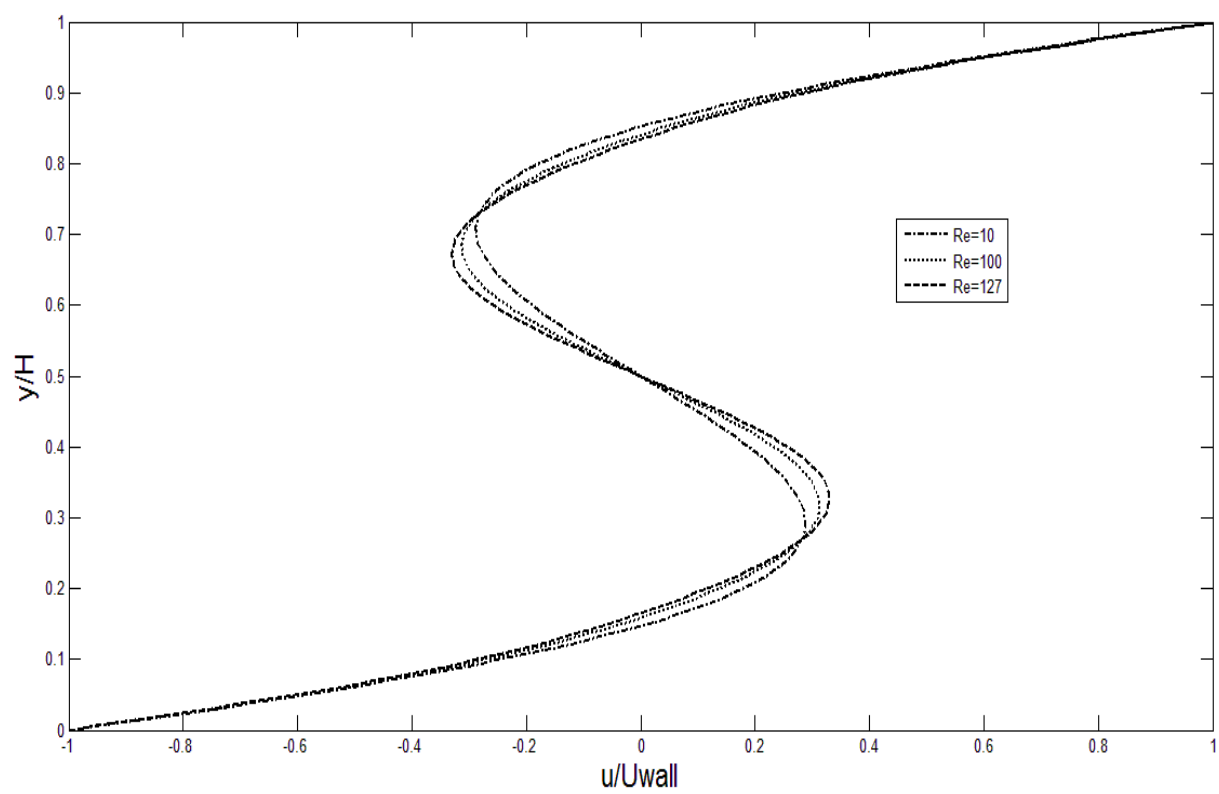

(b)

Figure 6. (a) Graph of v-velocity profile along $y=0.5$ and (b) graph of u-velocity profile along $\mathrm{x}=0.5$ inside cavity for different Reynolds numbers.

\section{CONCLUSIONS}

A four-sided lid-driven cavity was studied numerically by using the Adams-Bashforth scheme with a time splitting method. It shows that the vortices move nearer to the diagonal joining edge of the cavity as the Reynolds numbers increase. A comparison of the location of the centers of the vortices showed similar results. 


\section{ACKNOWLEDGMENTS}

The authors would like to thank the Universiti Teknologi Malaysia and Ministry of Higher Education, Malaysia for sponsoring this research. This research is supported by research grant Vot. 4F114 and 4L074.

\section{REFERENCES}

Alleborn, N., Raszillier, H., \& Durst, F. (1999). Lid-driven cavity with heat and mass transport. International Journal of Heat and Mass Transfer, 42(5), 833-853.

Boersma, B. J. (2011). A 6th order staggered compact finite difference method for the incompressible navier-stokes and scalar transport equations. Journal of Computational Physics, 230(12), 4940-4954.

Cadou, J. M., Guevel, Y., \& Girault, G. (2012). Numerical tools for the stability analysis of 2d flows: Application to the two-and four-sided lid-driven cavity. Fluid Dynamics Research, 44(3), 031403.

Cao, Z., \& Esmail, M. N. (1995). Numerical study on hydrodynamics of short-dwell paper coaters. AIChE Journal, 41(8), 1833-1842.

Choi, C.-Y., \& Balaras, E. (2009). A dual reciprocity boundary element formulation using the fractional step method for the incompressible navier-stokes equations. Engineering Analysis with Boundary Elements, 33(6), 741-749.

Gaskell, P., Summers, J., Thompson, H., \& Savage, M. (1996). Creeping flow analyses of free surface cavity flows. Theoretical and Computational Fluid Dynamics, $8(6), 415-433$.

Ghia, U., Ghia, K. N., \& Shin, C. (1982). High-re solutions for incompressible flow using the navier-stokes equations and a multigrid method. Journal of Computational Physics, 48(3), 387-411.

González, L., Ahmed, M., Kühnen, J., Kuhlmann, H., \& Theofilis, V. (2011). Threedimensional flow instability in a lid-driven isosceles triangular cavity. Journal of Fluid Mechanics, 675, 369-396.

Hansen, M. O., Sørensen, J. N., \& Shen, W. Z. (2003). Vorticity-velocity formulation of the $3 \mathrm{~d}$ navier-stokes equations in cylindrical co-ordinates. International Journal for Numerical Methods in Fluids, 41(1), 29-45.

Idris, M. S., Ammar, N. M. M., Tuan Ya, T. M. Y. S., \& Amin, A. M. (2013). Transient semi-circular lid-driven cavity flow using non- uniform structured grid method with upwind scheme. Journal of Mechanical Engineering and Sciences, 5, 713722.

Idris, M. S., Irwan, M. A. M., \& Ammar, N. M. M. (2012). Steady state vortex structure of lid driven flow inside shallow semi-ellipse cavity. Journal of Mechanical Engineering and Sciences, 2, 206-216.

Lo, D., Murugesan, K., \& Young, D. (2005). Numerical solution of three-dimensional velocity-vorticity navier-stokes equations by finite difference method. International Journal for Numerical Methods in Fluids, 47(12), 1469-1487.

Man, C., \& Tsai, C. W. (2008). A higher-order predictor-corrector scheme for twodimensional advection-diffusion equation. International Journal for Numerical Methods in Fluids, 56(4), 401-418.

Mendu, S. S., \& Das, P. (2012). Flow of power-law fluids in a cavity driven by the motion of two facing lids-a simulation by lattice boltzmann method. Journal of Non-Newtonian Fluid Mechanics, 175, 10-24. 
Musa, M. A., Abdullah, S., Azwadi, N., \& Zulkifli, R. (2011). Lattice boltzmann simulation of cavity flows at various reynolds numbers. International Review on Modelling \& Simulations, 4(4).

Oueslati, F., Ben Beya, B., \& Lili, T. (2011). Aspect ratio effects on three-dimensional incompressible flow in a two-sided non-facing lid-driven parallelepiped cavity. Comptes Rendus Mecanique, 339(10), 655-665.

Patil, D., Lakshmisha, K., \& Rogg, B. (2006). Lattice boltzmann simulation of liddriven flow in deep cavities. Computers \& Fluids, 35(10), 1116-1125.

Perot, J. B. (1993). An analysis of the fractional step method. Journal of Computational Physics, 108(1), 51-58.

Perumal, D. A., \& Dass, A. K. (2011). Multiplicity of steady solutions in twodimensional lid-driven cavity flows by lattice boltzmann method. Computers and Mathematics with Applications, 61, 3711-3721.

Rosdzimin, A. R. M., Zuhairi, S. M., \& Azwadi, C. S. N. (2010). Simulation of mixed convective heat transfer using lattice boltzmann method. International Journal of Automotive and Mechanical Engineering, 2, 130-143.

Sidik, N. A. C., \& Attarzadeh, S. M. R. (2011). An accurate numerical prediction of solid particle fluid flow in a lid-driven cavity. International Journal of Mechanics, 5(3), 123-128.

Wahba, E. M. (2009). Multiplicity of states for two-sided and four-sided lid driven cavity flows. Computers \& Fluids, 38(2), 247-253.

Yang, F., Shi, X., Guo, X., \& Sai, Q. (2012). Mrt lattice boltzmann schemes for high reynolds number flow in two-dimensional lid-driven semi-circular cavity. Energy Procedia, 16, 639-644.

Zhang, T., Shi, B., \& Chai, Z. (2010). Lattice boltzmann simulation of lid-driven flow in trapezoidal cavities. Computers \& Fluids, 39(10), 1977-1989. 